\title{
Research on the Design of Protective Clothing for Industrial Robots Assisted by CLO 3D
}

\author{
Jia-yi Sun ${ }^{\text {a }, ~ L u ~ C e n ~}{ }^{\text {a, Li-na Zhai }}{ }^{\text {a, }}{ }^{1}$, Gang Bai ${ }^{\text {b }}$ \\ a Zhejiang international institute of fashion technology, Zhejiang Sci-Tech University, \\ 310018, Hangzhou, Zhejiang, China \\ 'Shanghai Chunyu Automation Equipment Protection Technology Co., Ltd., 20043 \\ 7. Shanghai, China
}

\begin{abstract}
The development of intelligent industrialization has gradually increased the demand for industrial robots, and it has also promoted the emergence and development of industrial robot protective clothing. At this stage, the design methods of sewing patterns are limited to planes and lack simulation stitching and try-on in three-dimensional space. In this study, the virtual try-on of protective clothing for industrial robots was realized in the CLO3D environment, and the fabric was simulated using tools in the software. First, import the digital model of the industrial robot into CLO3D, and copy the AUTO CAD template in CLO3D. Then, input the results of the fabric test to simulate the fabric. Use the software's virtual stitching and virtual wearing to observe the wearing effect of protective clothing and the data-based fabric pressure, directly modify the model and observe the modification effect. After research, the three-dimensional simulated fitting can clearly show the characteristics of looseness or tightness and whether it is convenient to put on and take off. The model can be adjusted appropriately in time, which can reduce costs and improve production efficiency. It is an important exploration for the development of industrial robot protective clothing.
\end{abstract}

Keywords. Industrial robot, protective clothing, 3D fitting, fabric performance, CLO3D

\section{Introduction}

Made in China 2025 clearly classifies robots into ten core areas [1-2]. In recent years, the market demand for industrial robots has continued to expand [3]. Industrial robots often need to face harsh operating environments such as high temperature, acid, base, or sparks and static electricity, so they are often damaged, and their service life and work efficiency are severely limited [4]. Similar to human clothing, the manufacturing of protective clothing for industrial robots also requires the design of sewing patterns. However, the current stage of protective clothing takes a long time to sew and try on, resulting in low output efficiency and high cost. Usually, the clothing pattern making method is as follows: According to the digital model of the industrial robot, "frame 
selection" is performed from different perspectives such as front view, side view and top view in the two-dimensional view in the industrial CAD software [5]. After that, the designer makes inferences based on the three-dimensional modeling of the industrial robot in 3D modeling software, and directly draws the sewing patterns of the protective clothing on the digital model of the industrial robot.In the past, sewing patterns drawn by reasoning can only be evaluated by using real fabrics to make samples and try on them, and then the wearing effects can be evaluated and modified accordingly. Such a design and modification cycle time is long, which is not conducive to the improvement of production efficiency.By using CLO 3D software to simulate the three-dimensional stitching effect and wearing state of sewing patterns, the wearing effect can be quickly and directly evaluated and modified [6]. In addition, this research can provide a basis for the evaluation of the effect of industrial robots wearing protective clothing under the motion state, which is conducive to the development of more intelligent protective clothing design methods.

\section{Materials and Methods}

\subsection{Introduction to CLO 3D Software Features}

Nowadays, the popular virtual fitting software on the market mainly includes CLO 3D, Style 3D, Browzwear, etc. My Virtual Model, Fit-me.com and Fitiquette are also more mature virtual fitting platforms[7]. Among them, CLO 3D is one of the most commonly used virtual fitting software, featuring good fabric simulation performance. CLO 3D can form realistic effects by setting fabric attributes. Designers can change different physical effects including strength, diagonal tension, bending strength, deformation rate, deformation strength, density and coefficient of friction according to different attributes of the clothing. In addition, it is easy to operate and can be compatible with other platforms, especially clothing CAD platforms. This makes it possible to promote applications. It has powerful functions such as rendering, pattern editing and measurement. Using CLO3D, people can edit the plane model, or directly edit the threedimensional pattern worn on the human body. The 6th axis articulated industrial robot is currently the most representative robot in the production industry, and is widely used due to its excellent flexibility [8].

\subsection{Fabric Testing}

With the help of robot protective clothing manufacturers, we selected 6 different types of high temperature resistant fabrics for experiments. Perform basic physical tests, fabric bending stiffness performance test and fabric sample breaking strength performance test on 6 kinds of protective clothing fabrics, so as to obtain the experimental data of fabric weight, thickness, bending stiffness and breaking strength.

YG(B)022D automatic fabric stiffness tester is used to test the flexural rigidity of the fabric. The principle is to use a long and narrow fabric of $150 \mathrm{~mm} \times 25 \mathrm{~mm}$ as the cantilever beam. According to its flexibility, let it form an inclination angle of $41^{\circ}$ under the free end. According to the detection slope composed of the infrared beam instrument, the extension length, the bending resistance length and the bending rigidity of the test fabric at this time can be obtained.

The testing method for the breaking strength of protective clothing fabrics is as 
follows. Use the upper clamp of the electronic powerful machine to gently clamp one end of the $300 \mathrm{~mm} \times 90 \mathrm{~mm}$ experimental fabric, and then clamp the other end with the lower clamp to start the machine. The lower clamp will pull down at a certain speed until the tested fabric breaks, and record the breaking strength and breaking elongation. The breaking strength is the maximum force during the stretching process. The elongation at break refers to the elongation of the fabric when the tensile force is maximum. Knowing it and the initial elongation can calculate the elongation at break. Breaking strength and breaking elongation are related to the service life of the fabric.

\subsection{Computer-aided Design Method of Protective Clothing for Industrial Robots}

According to experimental data, fabrics with the best combined level of bending stiffness, breaking strength and elongation will be used to make protective clothing for industrial robots. The fabric test results will be input into the CLO3D software to complete the simulation of the fabric. The physical properties can be modified qualitatively and quantitatively in the fabric properties editing window.

After opening the digital model of the industrial robot with AUTOCAD software, the designer directly draws the basic sewing pattern in AUTOCAD, adds ease and designs special structures according to style requirements, and finally forms a dwj format template. Taking the shape, size and angle of the pattern in AUTOCAD as a reference, copy and draw the model in AUTOCAD in the model drawing area of CLO3D to obtain a protective clothing model that can be simulated and stitched. Use the simulated stitching function in CLO3D to watch the effect and make adjustments in the 3D clothing display area.

Finally, the garment is made according to the adjusted patterns. First, use a special protective thread to sew the partial structure, and then stitch each part as a whole. In addition, special structures such as velcro and elastic bands need to be stitched together.

\section{Results and Analysis}

\subsection{Fabric Test Results and Virtual Design of Fabrics}

\subsubsection{Fabric Test Results}

The following are the test results of fabric weight, thickness, bending stiffness, and breaking strength.

Table 1. Basic parameters of fabric samples

\begin{tabular}{ccc}
\hline Fabric Number & Weight $\mathbf{( g / \mathbf { m } ^ { \mathbf { 2 } } )}$ & Thickness $\mathbf{( m m})$ \\
\hline M1 & 672 & 0.624 \\
M2 & 717.33 & 0.639 \\
M3 & 653.33 & 0.578 \\
M4 & 557.33 & 0.554 \\
M5 & 546.67 & 0.552 \\
M6 & 394.67 & 0.457 \\
\hline
\end{tabular}

Table 2. Bending stiffness test of fabric samples

\begin{tabular}{cccc}
\hline Fabric Number & $\begin{array}{c}\text { Extention Length } \\
(\mathbf{m m})\end{array}$ & $\begin{array}{c}\text { Bending Length } \\
(\mathbf{m m})\end{array}$ & Bending Stiffness \\
\hline M1 & 133.8 & 66.9 & 2012 \\
M2 & 116.2 & 58.1 & 1406
\end{tabular}




\begin{tabular}{lrrr} 
M3 & 71.4 & 35.7 & 298 \\
M4 & 95.7 & 47.9 & 610 \\
M5 & 124.9 & 62.5 & 1331 \\
M6 & 71.8 & 35.9 & 182 \\
\hline
\end{tabular}

Table 3. Breaking strength performance test of fabric samples

\begin{tabular}{rrrrr}
\hline Fabric Number & $\begin{array}{c}\text { Breaking } \\
\text { Strength }(\mathbf{m m})\end{array}$ & $\begin{array}{c}\text { Elongation } \\
\text { variation }(\mathbf{m m})\end{array}$ & $\begin{array}{c}\text { Elongation } \\
\text { rate(\%) }\end{array}$ & Time (s) \\
\hline M1 & 1679.2 & 21.49 & 10.7 & 13 \\
M2 & 1974.3 & 36.87 & 18.4 & 22.3 \\
M3 & 1387.6 & 57.15 & 28.5 & 34.4 \\
M4 & 2568.2 & 22.37 & 11.1 & 13.5 \\
M5 & 1916.6 & 8.39 & 4.1 & 5.2 \\
M6 & 1918.8 & 67.24 & 33.6 & 40.5 \\
\hline
\end{tabular}

From Table 2 and Table 3, it can be found that fabric M1 has the highest bending stiffness index, so it is the most stiff and difficult to bend. It can maintain the stiffness of the overall garment during the operation of the industrial robot without collapsing and rubbing. The second to M1 bending stiffness is M2 and M5 fabrics, while the other three fabrics have weaker Bending stiffness. The test results of the breaking strength performance of the fabric sample show that the M4 fabric has the highest breaking strength and lower elongation. M6 fabric has the highest elongation, which can provide a greater degree of operation.

In summary, because M2 has a middle-to-upper level of bending stiffness among these fabrics, its breaking strength and elongation are also excellent, making it the best choice among the six fabrics. Its good bending stiffness can ensure that the manufactured protective clothing will not collapse, ensuring the aesthetics of the appearance and the inner space for movement. The high breaking strength and elongation of the fabric can ensure the long-term use of the protective clothing and the adaptability during exercise.

\subsubsection{Fabric Virtual Design in CLO3D}

In the fabric attribute editing window, the physical attributes of the fabric can be reset according to the parameters of the M2 fabric. The data of gram weight $717.33 \mathrm{~g} / \mathrm{m}^{2}$ and thickness $0.639 \mathrm{~mm}$ can be input accurately. However, the CLO3D software currently does not support the input of accurate data in terms of properties such as bending strength and deformation strength, but a draggable progress bar with a qualitative evaluation of 1-100 points is set. Therefore, in order to simulate the stiff characteristics of protective clothing fabrics, the weft strength, warp strength, bending strength weft and bending strength warp are all adjusted to 99 .

\subsection{CLO3D Structure Design and Modification}

First, in order to meet CLO3D's file format requirements, the digital model of the industrial robot needs to be converted to the obj format. After importing the robot 3D model that has been converted into obj format in the add virtual model interface in the CLO3D software, the original model needs to be deleted, leaving the $3 \mathrm{D}$ model of the 6th axis industrial robot. Select the corresponding line segment of the protective clothing patterns inside CLO3D for simulated stitching, and then wear it according to the positioning, you can get the following wearing effects, and carry out structural design and modification. 


\subsubsection{Base}

From the position of the pressure point of the patterns of the base in CLO3D, it can be seen that the opening of the patterns of the base is relatively narrow at the one-axis, which makes it difficult to wear. At the same time, the renderings show that the ease of the height direction of the base of the protective clothing is insufficient, causing a part of the fabric to contact the parts of the base. In this case, every time a shaft rotates, the fabric will be rubbed. If things go on like this, the fabric will be damaged to varying degrees. Therefore, the length of the sample in the height direction of the protective clothing should be lengthened. In addition, according to the CLO 3D measurement, the radius of the base opening should be expanded by about $3 \mathrm{~cm}$. From the overall aesthetics point of view, the ease parallel to the horizontal plane is larger, resulting in a loose wearing effect in protective clothing. Therefore, the ease given to both sides can be reduced to make the protective clothing more beautiful.
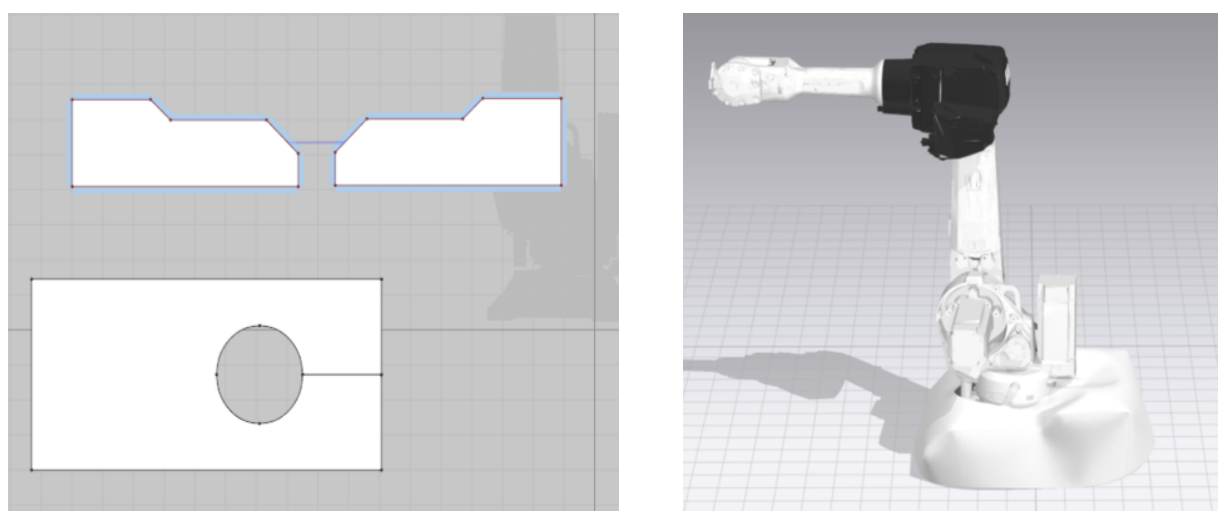

Figure 1. The sewing patterns and the try-on effect of the base

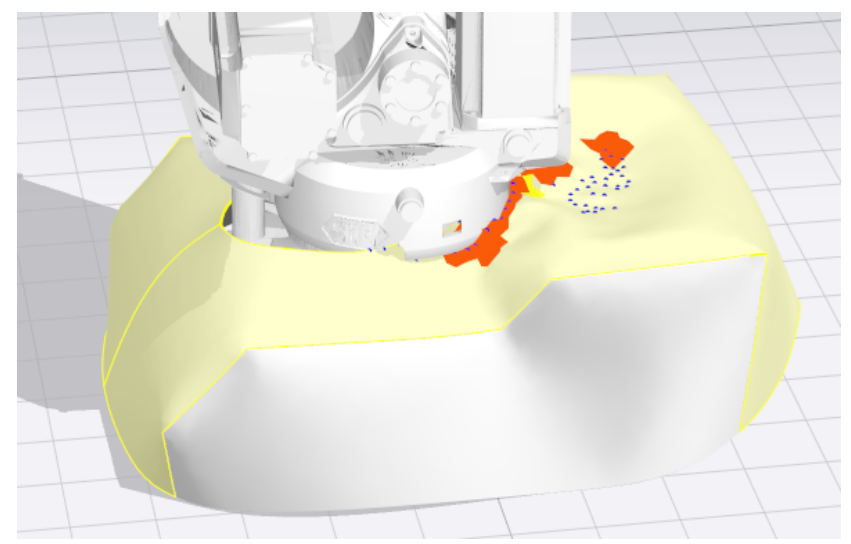

Figure 2. The effect of the base try-on

\subsubsection{1 st axis and 2 nd axis}

Figure 2 shows the wearing effect of the protective clothing that wraps the1st axis and 2nd axis. It can be seen that the bottom of the protective clothing has the same problem 
as the previous set of patterns, that is, the opening is too small to be worn. In addition, the small cube-shaped protective clothing that accommodates the protruding motor is also small in size, which creates pressure on the fabric. Therefore, the ease of protective clothing covering the motor part should be made larger. Therefore, the radius of the opening circle at the bottom of the 1 st axis should be increased by $3 \mathrm{~cm}$, and the side length of the 2 nd axis cube should be increased by $1 \mathrm{~cm}$.

\subsubsection{4th axis, 5th axis and 6th axis}

Observing the $3 \mathrm{~d}$ renderings, it can be seen that due to the larger ease of the 4th axis patterns, the robot wears relatively loosely. The ease can be appropriately reduced, and the length of the patterns can be reduced by $2 \mathrm{~cm}$. Finally, the fan-shaped patterns are redrawn according to the reduced ease and length. As shown in Figure 3, the fit between the protective clothing and the 4th axis and 5th axis is not enough, and there is a relatively large gap between the protective clothing and the industrial robot, which causes the protective clothing to appear loose at the 5th axis. Therefore, it is necessary to reduce the ease here and control the size of the opening at the connection, so as to make the whole structure more beautiful and streamlined. The part can be redrawn based on the 4th axis data.

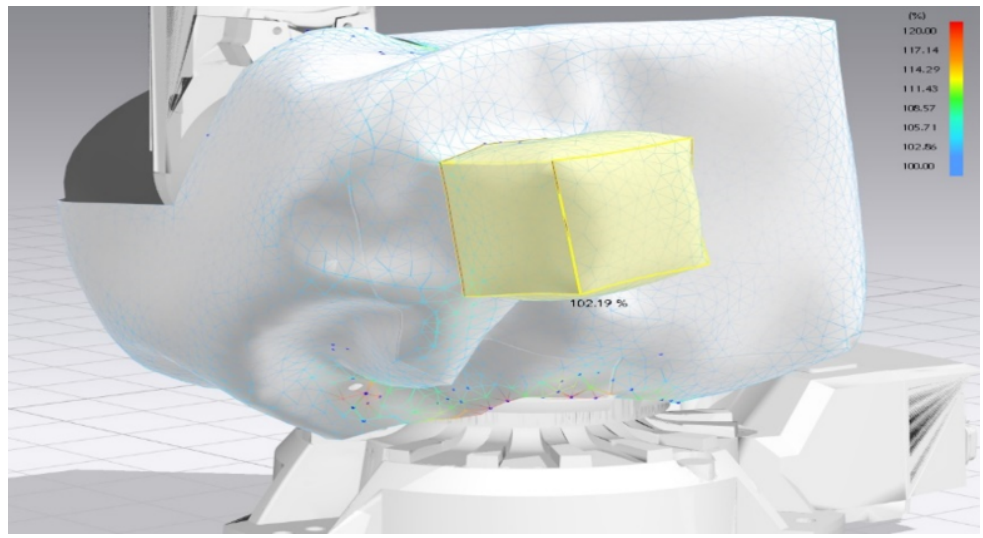

Figure 3. Try-on and pressure diagram between the first and second axis

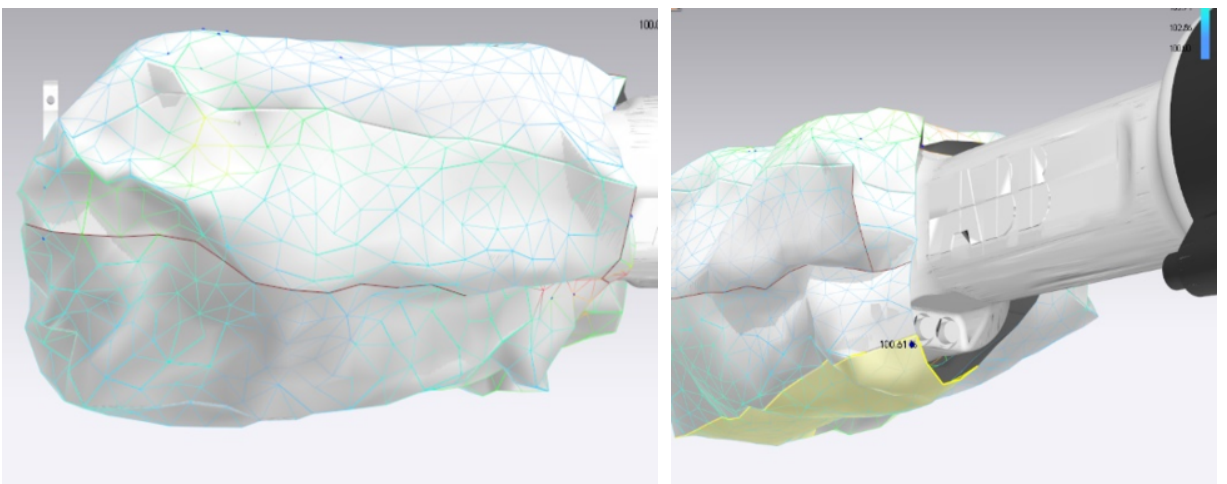

Figure 4. Five and six axis try-on 


\subsection{Final Patterns and Ready-to-wear}

In the 3D simulation effect interface in 3DCLO, the marks made on the 3D simulation dressing drawing with the brush can be displayed in the patterns, so that the sewing and dressing effects and the modification of the patterns can be carried out at the same time, which greatly improves the efficiency of debugging the suitability of the patterns. Properly increasing the opening of the base of the protective clothing and the opening at the lower part of the one-axis and two-axis can not only make wearing more convenient, but also effectively prevent the wear caused by frequent rotation of the one-axis.In addition, the ease of protective clothing patterns that accommodate special structures such as motors has also been appropriately adjusted to make it more convenient to wear. For the parts where the protective clothing is too loose as shown by the three-dimensional simulation wearing effect, reducing the amount of ease can effectively reduce the appearance of bubbles, improving the efficiency of fabric utilization and the aesthetics of the protective clothing. Through the simulation and real-time modification and adjustment of the protective clothing wearing effect, the protective clothing patterns have been greatly improved in terms of ease adjustment, opening size, and tightness in a short period of time.

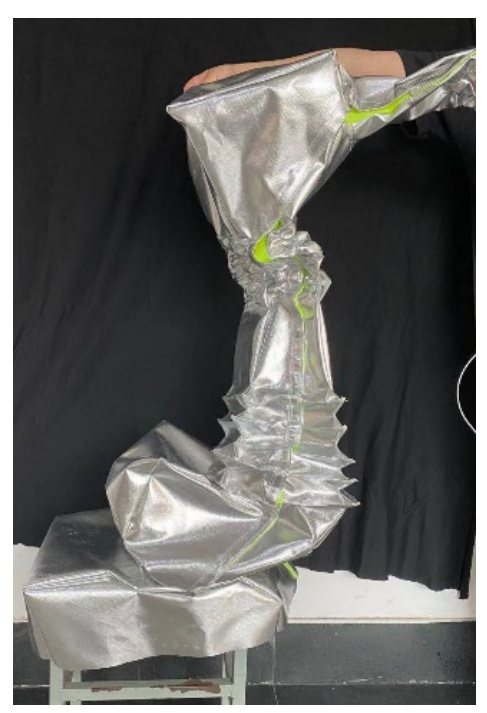

Figure 5. The samples made from modified patterns

\section{Conclusion}

It is feasible to use CLO3D software to assist the design and development of industrial robot protective clothing. Compared with the traditional design method of industrial robot protective clothing, the design method of using CLO3D auxiliary patterns has the advantages of turning abstraction into concrete, improving efficiency and saving cost. The design of traditional industrial protective clothing is limited to a two-dimensional plane. However, the design of protective clothing for industrial robots assisted by CLO3D connects the gaps of patterns and three-dimensional fittings, which effectively saves the cost and cycle of sewing and repeated modification of samples. CLO 3D can 
simulate the properties of fabrics, basic information such as weight and thickness can be accurately entered, and other properties such as bending strength can also be effectively simulated. The fabric pressure display in the software allows people to visually observe the fabric pressure state that was invisible to the naked eye in the past, thereby helping designers avoid structural defects before putting the protective clothing into practical application.

The future CLO3D-assisted industrial robot protective clothing design has greater development potential. First of all, the measurement method of fabric properties at this stage is qualitative, and with the development of technology in the future, it is possible to achieve accurate numerical entry and increase the accuracy of simulation. At the same time, the more complicated structure of the protective clothing of industrial robots may also realize rapid simulated stitching in the future. In addition, the current threedimensional simulation is still a simulation in a static state. If effective joint motion can be achieved, it is of great significance for observing and improving the mobility of industrial robot protective clothing.

\section{References}

[1] Zeng ZK, Talking about the debugging skills of industrial robots in the "intelligent manufacturing" project, Technology and Economic Guide 29 (20), 32-33.

[2] Equipment Industry Department of the Ministry of Industry and Information Technology. "Made in China 2025 " promotes the development of robots, Robot Technique and Application 2015(03), 31-33.

[3] Lu PY, Interpretation of the "White Paper on the Development of China's Robot Industry (2016 Edition)", Robot Industry, 2016(03), s26-32.

[4] Chi Z, Research on the Standard System of Protective Clothing for Industrial Robots, [D]. Donghua University, 2018.

[5] Chen YS, Bai G, Yan WM. Design and development of protective clothing for industrial robots, Shanghai Textile Science \& Technology 43(01):44-45+60.

[6] Lee N, Sung OK, and Sookjin K.The Design Development of Family Formal Clothes Using 3D Virtual Clothing Software. Journal of Fashion Business 23.3(2019)

[7] Ni A, Comparative analysis of the implementation methods of virtual fitting technology in online stores, Journal of Silk 51(02):40-46.

[8] Zhou C, Research and design of protective clothing based on six-axis articulated industrial robot, [D]. Donghua University, 2017. 